\title{
Dynamic Simulations in Cost and Time Estimation of the Construction Process
}

\author{
V. Beran, E. Hromada
}

This paper describes a model which is able to simulate the costs and the duration of construction for a building project. The model predicts the set of expected costs and the duration of the project depending on input parameters such as production rate, scope of the work, the time schedule, bonding conditions, maximum and minimum deviations from the scope of the work, and the production rate. Clients are able to make proper decisions concerning the time and cost schedules of their investments.

Keywords: simulation, time scheduling, cost scheduling, reliability and risk.

\section{Introduction}

A simulation of construction activities on the basis of production rate makes it possible to monitor the reliability of the expected time schedule and the total costs. The input parameters are production rate, scope of the work, the time schedule, bonding conditions, maximum and minimum deviations from the scope of the work, and the production rate.

The simulated model can be used at many levels of project management. Clients are able to make decisions about implementing their intentions; competitors can assess a bid price; building contractors can make a detailed calculation of the costs and the time schedule of construction activities, and they can optimize the construction process.

The simulation model stems form the research of Haas, Hajek [4] and then Beran, Dlask [1] and [2], which has been carried out at the Faculty of Civil Engineering, Czech Technical University in Prague during the last ten years.

\section{Definition of the problem}

Within the framework of initiating a simulation of a building project, it is necessary to define the problem as such. The application obtains input data by means of the Module of input data, which defines the particular construction activities, and the volume $\boldsymbol{Q}$ of these particular construction activities is expressed in physical or financial units, its production rate $\mathrm{v}$ and bonding conditions $\therefore \boldsymbol{D}_{\text {connection activities }}$ linking particular activities. $\boldsymbol{T} \boldsymbol{A} \boldsymbol{B}_{\text {project }}$ characterizes the calculation as a meta problem called Dynamic progress chart (flow-sheet). $N$ generally characterizes the sequential networks $N_{i}$ [2]. The set expression is as follows:

$\boldsymbol{T A} \boldsymbol{B}_{\text {project }}=$

$\left\{N_{i} \mid\left[\boldsymbol{D}=f\left(\boldsymbol{Q} \mid\right.\right.\right.$ risk, $\boldsymbol{v} \mid$ risk, $\left.\left.\left.\therefore \boldsymbol{D}_{\text {connection activities }}\right)\right], i=1, \ldots\right\}$,

where $i$ are partial processes and $\boldsymbol{D}$ is the set of activity durations, while risk influence is a conditioned externality (see Ta-

Table 1: Dynamic progress chart of a building project

\begin{tabular}{|c|c|c|c|c|c|c|c|c|c|c|c|c|c|c|c|c|c|c|c|c|c|c|c|c|c|c|c|c|c|c|}
\hline \multirow{2}{*}{ Activity } & \multirow{2}{*}{\begin{tabular}{|c|}
$\begin{array}{c}\text { Scope } \\
\text { of work }\end{array}$ \\
\end{tabular}} & \multirow{2}{*}{\begin{tabular}{|c|}
$\begin{array}{c}\text { Production } \\
\text { speed }\end{array}$ \\
\end{tabular}} & \multirow{2}{*}{\begin{tabular}{|c|}
$\begin{array}{c}\text { Time } \\
\text { duration }\end{array}$ \\
\end{tabular}} & \multirow{2}{*}{ Start } & \multirow{2}{*}{ End } & \multicolumn{25}{|c|}{ Day } \\
\hline & & & & & & 1 & \begin{tabular}{|l|}
2 \\
\end{tabular} & \begin{tabular}{|l|}
3 \\
\end{tabular} & \begin{tabular}{|l|}
4 \\
\end{tabular} & \begin{tabular}{|l|}
5 \\
\end{tabular} & \begin{tabular}{|l|l|}
6 \\
\end{tabular} & \begin{tabular}{|l|}
7 \\
\end{tabular} & \begin{tabular}{|l|}
8 \\
\end{tabular} & \begin{tabular}{|l|}
9 \\
\end{tabular} & 10 & 11 & \begin{tabular}{|l|}
12 \\
\end{tabular} & \begin{tabular}{|l|}
13 \\
\end{tabular} & \begin{tabular}{|l|}
14 \\
\end{tabular} & 15 & \begin{tabular}{|l|}
16 \\
\end{tabular} & \begin{tabular}{|l|}
17 \\
\end{tabular} & \begin{tabular}{|l|}
18 \\
\end{tabular} & 19 & 20 & \begin{tabular}{|l|}
21 \\
\end{tabular} & 22 & 23 & 24 & 25 \\
\hline Ground works & 150 & 33 & 5 & 2 & 6 & $\ldots$ & 33 & 33 & 33 & 33 & \begin{tabular}{|l|}
19 \\
\end{tabular} & & & & & & & & & & & & & & & & & & & \\
\hline Water connection & 191 & 23 & 10 & 8 & 17 & & & & & & & ... & 23 & 22 & 22 & \begin{tabular}{|l|}
21 \\
\end{tabular} & \begin{tabular}{|l|}
20 \\
\end{tabular} & \begin{tabular}{|l|}
19 \\
\end{tabular} & \begin{tabular}{|l|}
19 \\
\end{tabular} & \begin{tabular}{|l|}
18 \\
\end{tabular} & \begin{tabular}{|l|}
17 \\
\end{tabular} & \begin{tabular}{|l|}
10 \\
\end{tabular} & & & & & & & & \\
\hline Sewerage connection & 207 & 55 & 4 & 9 & 12 & & & & & & & & & 55 & \begin{tabular}{|l|}
64 \\
\end{tabular} & 74 & \begin{tabular}{|l|}
15 \\
\end{tabular} & & & & & & & & & & & & & \\
\hline Electricity connection & 93 & 93 & 1 & 19 & 19 & & & & & & & & & & & & & & & & & & & 93 & & & & & & \\
\hline Bottom construction & 456 & 36 & 13 & 13 & 25 & & & & & & & & & & & & & \begin{tabular}{|l|}
36 \\
\end{tabular} & \begin{tabular}{|l|}
36 \\
\end{tabular} & \begin{tabular}{|l|}
37 \\
\end{tabular} & \begin{tabular}{|l|}
37 \\
\end{tabular} & \begin{tabular}{|l|}
37 \\
\end{tabular} & \begin{tabular}{|l|}
38 \\
\end{tabular} & 38 & \begin{tabular}{|l|}
38 \\
\end{tabular} & \begin{tabular}{|l|}
39 \\
\end{tabular} & \begin{tabular}{|l|}
39 \\
\end{tabular} & 40 & 40 & 1 \\
\hline Dumping place & 50 & 50 & 1 & 4 & 4 & & & & 50 & & & & & & & & & & & & & & & & & & & & & \\
\hline Overhead construction & 1010 & 42 & 14 & 26 & 39 & & & & & & & & & & & & & & & & & & & & & & & & & \\
\hline Roof & 308 & 48 & 5 & 41 & 45 & & & & & & & & & & & & & & & & & & & & & & & & & \\
\hline Inside parget & 66 & 32 & 3 & 47 & 49 & & & & & & & & & & & & & & & & & & & & & & & & & \\
\hline Facade rendering & 63 & 30 & 3 & 51 & 53 & & & & & & & & & & & & & & & & & & & & & & & & & \\
\hline Completing works & 40 & 7 & 6 & 50 & 55 & & & & & & & & & & & & & & & & & & & & & & & & & \\
\hline \multicolumn{6}{|c|}{ Demands on resources through time $Q^{\prime}(t)$} & 0 & ల్ల & ஜ & ஜ & ల్ల & $\stackrel{2}{\div}$ & 0 & กี & F & ఋ & ڤ & ю & 논 & 员 & 논 & ज्ञ & $\stackrel{9}{q}$ & $\mathscr{m}$ & 萧 & $\mathscr{m}$ & gి & g्ल & 웅 & 요 & - \\
\hline \multicolumn{6}{|c|}{ Total demands on resources through time $Q(t)$} & 0 & ల్ల & $\because$ & $\stackrel{g}{g}$ & 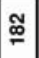 & ฉి & ฉ્సે & สี & $\overline{\bar{\nu}}$ & $\mathscr{् ్}$ & $\overline{\mathbf{g}}$ & $\frac{0}{6}$ & $\overline{6}$ & జ్ํ & :्్̈ & ผ & జ & జ్ఞ & $\overline{5}$ & \% & జ్ำ & $\underset{\square}{\mathscr{Z}}$ & 인 & 吉 & $\stackrel{\mathscr{g}}{\stackrel{9}{\leftarrow}}$ \\
\hline
\end{tabular}

Key:

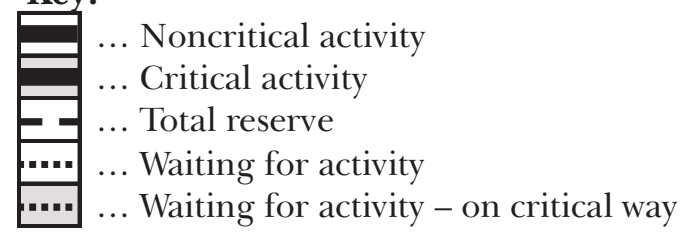


ble 1). The notation is supplement by conditionality of the breach of the assumed input parameters - the scope of the work and the production rate.

A practical solution of the calculation according to the dynamic progress chart (1) is based on inputting the work volume, the production rate and a time schedule of the particular activities. The time duration in the dynamic progress chart is calculated as the quotient of quantities $\boldsymbol{Q}$ and $\boldsymbol{v}$, or more precisely $D_{i}=Q_{i} / v_{i}$. The input data included in the Module of input data in the Connection activities sheet defines the bonding conditions among the particular production activities. The Deviations of project parameters 1 and 2 sheet contain input data about the minimum / maximum deviations of the scope of the work and the production rate of the particular activities, based on the expected parameters of the building process [6].

\section{Solution and an example}

On the basis of the Excel VBA application, the algorithm enable us to calculate an instant dynamic progress chart of the building project, including a time schedule of resources in terms of expression (1), the dynamic progress chart is differences calculated on the basis of a common progress chart. The calculation is based on the production rates and the individual activities, which are described in columns Start and End (Tab. 1), which present the links between the individual activities. It practically represents the relations between the declared function $f\left(\boldsymbol{Q} \mid\right.$ risk, $\boldsymbol{v} \mid$ risk, $\left.\therefore \boldsymbol{D}_{\text {connection activities }}\right)$ from expression (1) and the composition of the task as a consecutive process on the basis of the time duration of the individual processes $N[\boldsymbol{D}][1,2,5]$.

The dynamic progress chart creates a comprehensive methodically uniform model. The outputs of the model include information about the deadlines for the start and the end of the production activities, and information about cost schedules. The application creates a graphic visualization of the demand for resources in time (see Fig. 1).

The question of continuity of the project realization is interconnected with the cost-cutting management measures.
The varying construction rate causes changes in construction costs. The flow of the construction costs is a significant indicator of the economy of the capital employment.

The calculation and software application described here can be used for evaluating of bid proposals for investment projects. The approach carries out a two-dimensional simulation. The project described in propositions time and costs will be marked as a predefined project. The discrete probabilistic variables $(T ; C)$ obtain the values $\left(t_{i} ; c_{j}\right)$. We mark it as follows: $\mathbf{P}\left(T=t_{i} ; C=c_{i}\right)=p_{i j}$.

The SW application simulates the presumed development of the examined construction phase, the whole construction project or just a set of construction activities. We can identify the effects of changes and we can view management changes in the scope of particular jobs (construction activities) and the probability (reliability) of meeting the proposed (contracted) completion deadline $t_{\text {fin }}$ and the proposed contracting cost $\operatorname{limit} c_{\text {fin }}$. In general we are searching for acceptable

$F\left(t_{\text {fin }} ; c_{\text {fin }}\right)=\sum_{t} \sum_{c} \mathbf{P}\left(T<t_{\text {fin }} ; C<c_{\text {fin }}\right)$

for the selected project activities $A_{k}$

or their activity sets $A_{k}, A_{\mathrm{l}}, \ldots, A_{x}$, functionality-designed into a network.

The results of particular simulations

$$
\mathbf{P}(T ; C)=\mathbf{P}\left(\operatorname{sim} \boldsymbol{T} \boldsymbol{A} \boldsymbol{B}_{\text {project }}(T ; C)\right)
$$

for the example described below are $T \in\langle 45 ; 89\rangle$ and $C \in\langle 2411 ; 3131\rangle$. Simulation data is continuously recorded on the basis of (1), [2]. The simulation is based on the time schedule given in Table 1. 50000 simulations can be ranged into $30 \times 30$ categories. When a simulation is finished, the recorded data serves as a basis for a statistical analysis of the construction processes. The data file serves for the final analysis and the inter alia is a basis for modified $3 \mathrm{D}$ visualization, as shown in Fig. 2. The calculation of the expected or fixed probability starts, ends and reserves the results, as shown in Fig. 3 and Fig. 4.
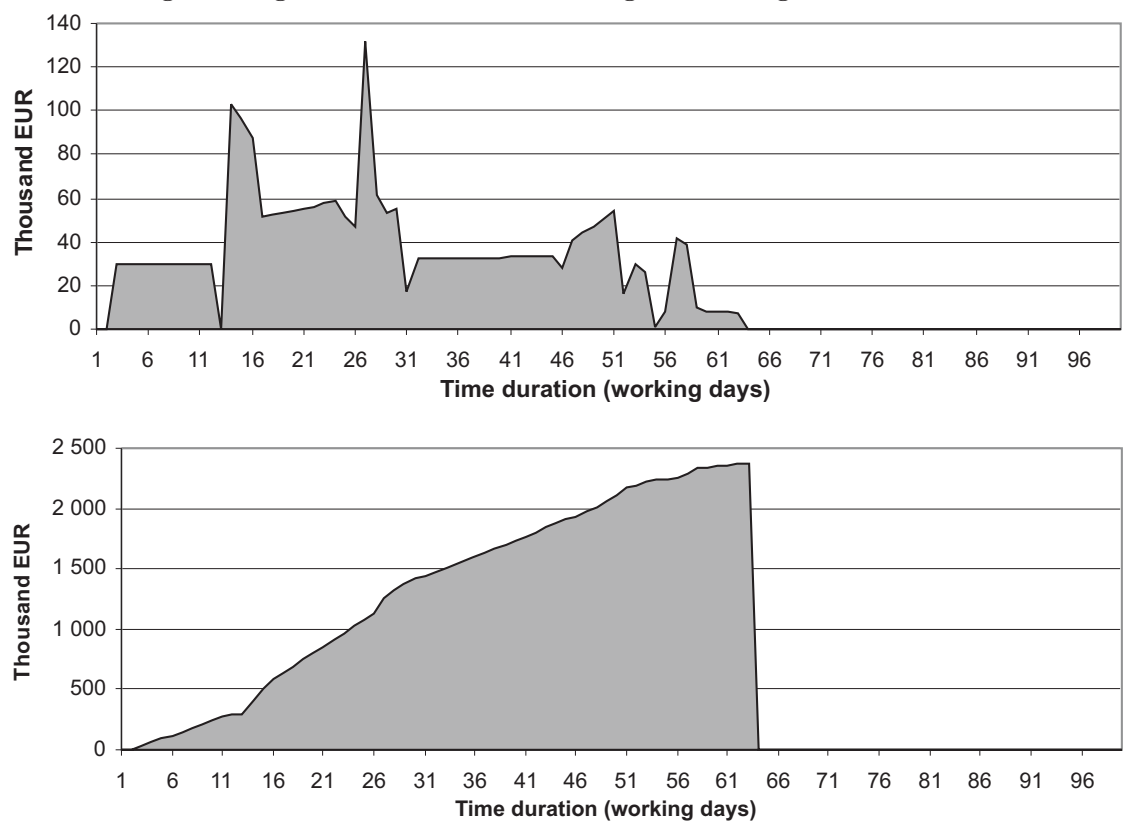

Fig. 1: Required cash flow of capital needed in the course of the construction period and the cumulative need for capital 

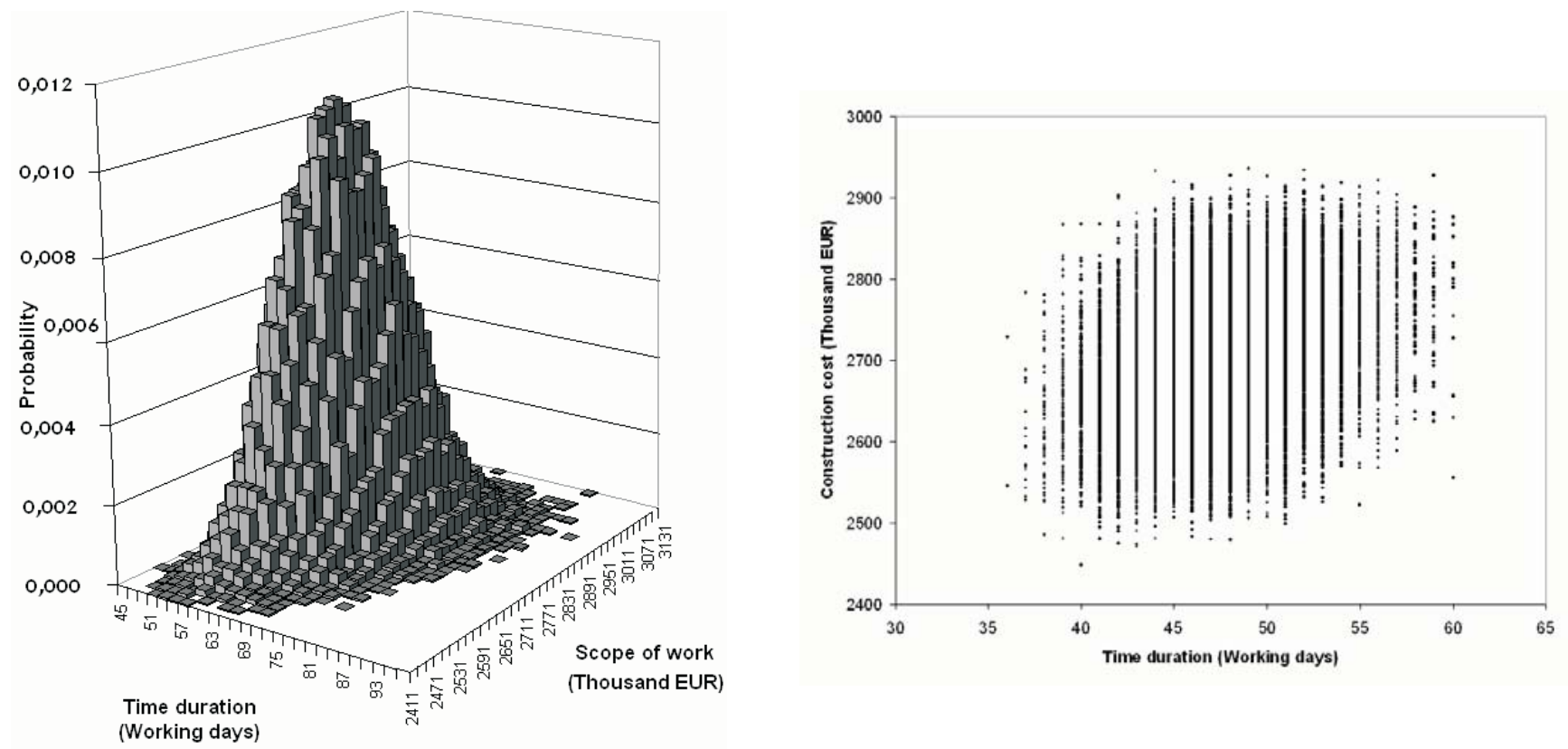

Fig. 2: Example of a 3D probability bar chart as an expression on the basis of (1)

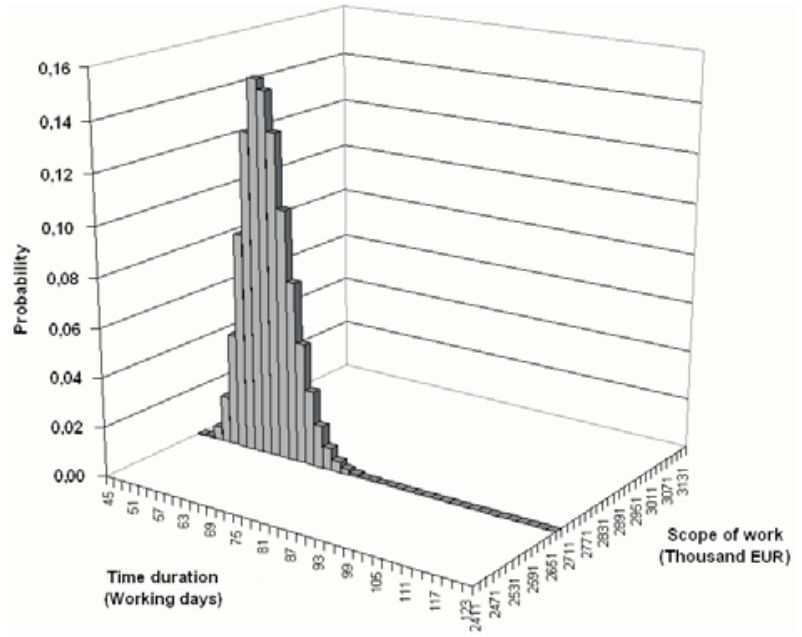

Fig. 3: Probability 3D bar chart for a construction project with fixed cost scope $C$

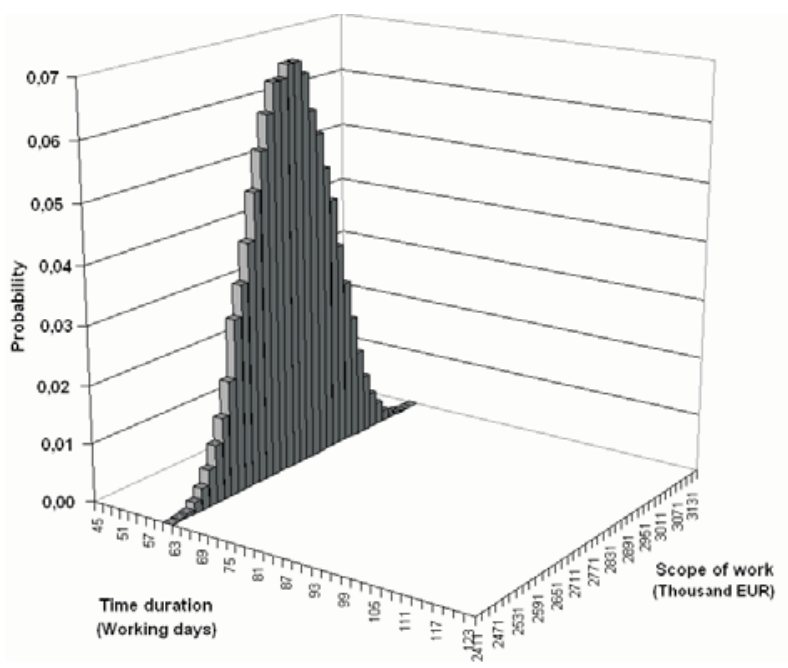

Fig. 4: Probability 3D bar chart for a construction project with fixed time duration $T$
Table 2 contains structural data of the comprehensive simulation example. The particular items are calculated as a construction bid proposal which is further described by a simulation study. This shows how practically competitive and realistic the supposed completion date and costs are.

$$
\begin{aligned}
& \mathbf{P}\left(T_{(45,89)} ; C_{(2411,3131)}\right)=\mathbf{P}\left(\operatorname{sim} \boldsymbol{T} \boldsymbol{A} \boldsymbol{B}_{\text {project }}(T ; C)\right)= \\
& \mathbf{P}\left(\operatorname { s i m } \left\{N_{i} \mid\left[\boldsymbol{D}=f\left(\boldsymbol{Q} \mid \text { risk, v|risk, } \therefore \boldsymbol{D}_{\text {connection activities }}\right)\right],\right.\right.
\end{aligned}
$$

for subprojects or subactivities $i=1, \ldots\}$ ).

The construction project [3] is proposed with the time schedule and the scope of the work as given in the 3D bar chart. The ellipse in Table 2 shows the shift of probability in time and costs. Using this approach, he results of the simulations can be specified more precisely. The occurrence frequencies of the particular scenarios of building project bids are comparable. The highest simulation frequency values in the 3D bar graph indicate the highest probabilities of potential success scenarios for the construction project. In the given case, the building project will be completed with satisfactory commercial probability, within the range of 57 to 59 days, and the construction cost will be in the range of 2731 to 2751 thousand EUR.

Within the framework a building project simulation, the calculation frequently reveals a unique regular solution. An example of a 3D probability bar chart with a unique regular solution is shown in Fig. 2.

In the case of complicated bonding conditions and other additional interdependences between the particular activities, the solution of the simulation may not be unique.

Fig. 5 and Fig. 5 a present a building project in which the input parameters contain a specific interdependence within the first activity (ground works). Let us compare these results with Fig. 2. In the event that the first activity takes more than 25 days, the building ground machinery must without delay be removed to another major activity (another building project). This situation causes slippage of dates within the range 
Table 2: The example of a 3D bar chart illustrating the result of 50000 simulations

\begin{tabular}{|c|c|c|c|c|c|c|c|c|c|c|c|c|c|c|c|c|c|c|c|c|c|c|c|c|c|c|c|c|c|c|c|c|c|c|c|c|c|c|c|}
\hline \multirow{2}{*}{\multicolumn{2}{|c|}{ Classes }} & \multicolumn{37}{|c|}{ Scope of work (Thousand EUR) } & \multirow[b]{2}{*}{ Total } \\
\hline & & 景 & 商 & 帘 & $\underset{\mathrm{S}}{\mathbf{5}}$ & $\stackrel{\bar{s}}{\mathbf{s}}$ & 突 & 蒿 & 空 & $\bar{\Sigma}$ & 恿 & 空 & 産 & 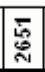 & \begin{tabular}{|l|} 
\\
i \\
N
\end{tabular} & 悥 & $\underset{\mathbf{N}}{\mathbf{N}}$ & 啇 & 商 & $\overline{\mathbf{E}}$ & ì & 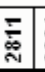 & 总 & 咇 & 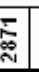 & 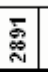 & 홍 & 寅 & 댕 & 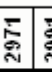 & 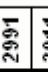 & 항 & 商 & Бृ & 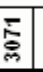 & હ্户 & 풀 & - & \\
\hline \multirow{23}{*}{ 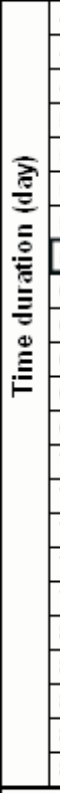 } & 45 & & & & & 1 & & & 4 & & & & & & \begin{tabular}{|l|l}
1 \\
\end{tabular} & & & 1 & & & 1 & & & & & & & & & & & & & & & & & & 5 \\
\hline & 47 & & & & & 2 & 4 & 1 & 1 & 5 & 1 & 2 & 1 & 1 & 2 & 2 & 1 & 1 & & & & & & & 2 & & & & & & & & & & & & & & 26 \\
\hline & 49 & & 1 & 2 & 4 & 6 & 10 & 6 & 14 & 17 & 17 & 19 & 18 & 25 & 18 & 20 & 21 & 14 & 8 & 13 & 7 & 5 & 5 & 3 & 2 & 1 & 1 & & & & & & & & & & & & 58 \\
\hline & 51 & 1 & 3 & 5 & 8 & 19 & 23 & 3 & 42 & 42 & 68 & 68 & 71 & 74 & 69 & 81 & \begin{tabular}{l|l}
67 & \\
\end{tabular} & 41 & 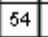 & 60 & 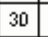 & 311 & 19 & 10 & 7 & 9 & 2 & & 1 & 2 & & & & & & & & & 40 \\
\hline & 53 & 2 & 3 & 6 & 10 & 24 & 41 & 64 & 81 & 101 & 138 & 136 & 157 & 180 & 170 & 191 & \begin{tabular}{l|l}
158 & 1 \\
1
\end{tabular} & 145 & 122 & 94 & 113 & 84 & 86 & \begin{tabular}{l|l}
56 & \\
\end{tabular} & \begin{tabular}{|l|l}
43 \\
\end{tabular} & \begin{tabular}{|l|}
30 \\
\end{tabular} & 16 & 8 & 8 & & 2 & & 1 & & & & & & 270 \\
\hline & \begin{tabular}{|l|}
55 \\
\end{tabular} & 1 & 5 & 2 & 13 & 34 & \begin{tabular}{l|l}
53 & \\
\end{tabular} & 7 & 110 & 135 & 201 & 224 & 268 & 313 & 317 & 336 & 3363 & 307 & 287 & 267 & \begin{tabular}{ll|}
253 & 2 \\
\end{tabular} & $\begin{array}{ll}205 & 1 \\
\end{array}$ & \begin{tabular}{l|l}
177 & 1 \\
\end{tabular} & \begin{tabular}{l|l}
127 & 1 \\
\end{tabular} & \begin{tabular}{l|l}
127 \\
\end{tabular} & \begin{tabular}{|l|}
75 \\
\end{tabular} & \begin{tabular}{l|l|}
56 \\
\end{tabular} & \begin{tabular}{l|l}
34 \\
\end{tabular} & 23 & 10 & 6 & 5 & & & 2 & & & & 386 \\
\hline & \begin{tabular}{|l|l|}
57 \\
\end{tabular} & 1 & & 8 & 20 & 26 & \begin{tabular}{l|l|}
63 & \\
\end{tabular} & 8 & 1 & \begin{tabular}{|l|l}
182 \\
\end{tabular} & 240 & 299 & 351 & 3720 & 446 & 450 & $454 \mid 4$ & 478 & \begin{tabular}{|l|}
477 \\
\end{tabular} & 458 & $383=$ & $341-3$ & 2812 & \begin{tabular}{ll|l}
263 & 2 \\
\end{tabular} & 205 & 141 & 111 & \begin{tabular}{l|l|}
87 & \\
\end{tabular} & \begin{tabular}{l|l}
52 & \\
\end{tabular} & \begin{tabular}{l|l}
26 & 2 \\
\end{tabular} & 23 & 9 & 3 & 2 & 1 & & & & 457 \\
\hline & \begin{tabular}{|l|}
59 \\
\end{tabular} & & 1 & 2 & 12 & 28 & \begin{tabular}{l|l}
46 & \\
\end{tabular} & 87 & 110 & 156 & 245 & 298 & 342 & 421 & 433 & 480 & $\begin{array}{l}535 \\
5\end{array}$ & \begin{tabular}{l|l|}
543 \\
\end{tabular} & \begin{tabular}{|l|l|}
552 \\
\end{tabular} & 542 & 469 & $\begin{array}{lll}402 & 3 \\
\end{array}$ & $397 / 3$ & उ5ा & 204 & 250 & \begin{tabular}{l|l}
174 & 1 \\
\end{tabular} & 129 & \begin{tabular}{l|l}
92 \\
\end{tabular} & \begin{tabular}{l|l}
58 & 3 \\
\end{tabular} & 31 & \begin{tabular}{|l|l|}
17 & \\
\end{tabular} & 10 & 6 & 2 & 1 & & & 516 \\
\hline & 61 & & & 4 & \begin{tabular}{|l|}
9 \\
\end{tabular} & 21 & \begin{tabular}{l|l}
33 & \\
\end{tabular} & 50 & 98 & 140 & 212 & 257 & 313 & 319 & \begin{tabular}{|l|}
410 \\
\end{tabular} & 491 & 514 & 485 & 522 & 515 & \begin{tabular}{|l|}
520 \\
\end{tabular} & 4804 & 4343 & $\begin{array}{ll}382 & 2 \\
\end{array}$ & 298 & 252 & \begin{tabular}{l|l}
197 \\
\end{tabular} & 141 & \begin{tabular}{|l|l|}
87 \\
\end{tabular} & \begin{tabular}{l|l}
60 & 2 \\
\end{tabular} & \begin{tabular}{l|l}
28 & 2 \\
\end{tabular} & 22 & 10 & 3 & 1 & 2 & & & 310 \\
\hline & 63 & & 1 & 1 & \begin{tabular}{|l|}
8 \\
\end{tabular} & 10 & \begin{tabular}{l|l}
23 & \\
\end{tabular} & 47 & 56 & \begin{tabular}{|l|}
93 \\
\end{tabular} & 137 & 178 & 230 & 279 & 355 & 378 & \begin{tabular}{l|l}
465 & 4 \\
\end{tabular} & 447 & 445 & \begin{tabular}{l|l}
462 \\
48
\end{tabular} & 480 & 4153 & 3833 & $\begin{array}{l}3483 \\
\end{array}$ & 315 & 278 & 22) 1 & 1701 & \begin{tabular}{l|l}
112 \\
\end{tabular} & \begin{tabular}{l|l}
83 & 5 \\
\end{tabular} & \begin{tabular}{l|l}
53 & 3 \\
\end{tabular} & \begin{tabular}{l|}
32 \\
2
\end{tabular} & 15 & $2^{2}$ & 2 & & & 1 & 6532 \\
\hline & 65 & & 1 & & \begin{tabular}{|l|l|}
6 \\
\end{tabular} & 8 & 10 & 22 & 40 & \begin{tabular}{|l|l|}
56 \\
\end{tabular} & 106 & 137 & 130 & 10 & 259 & 251 & $\begin{array}{lll}308 & 3 \\
\end{array}$ & 341: & \begin{tabular}{|l|l}
364 & \\
\end{tabular} & 351 & \begin{tabular}{ll|}
374 & 3 \\
\end{tabular} & $\begin{array}{lll}353 & 3 \\
\end{array}$ & 3342 & \begin{tabular}{l|l}
290 & 2 \\
\end{tabular} & \begin{tabular}{l|l}
277 & 2 \\
\end{tabular} & 245 & 1681 & 154 & \begin{tabular}{l|l}
97 \\
\end{tabular} & \begin{tabular}{l|l}
65 & 4 \\
\end{tabular} & \begin{tabular}{l|l}
41 & 2 \\
\end{tabular} & 22 & 13 & 12 & 3 & 1 & & & 5049 \\
\hline & \begin{tabular}{|l|}
67 \\
\end{tabular} & & & 1 & \begin{tabular}{|l|}
3 \\
\end{tabular} & 3 & 4 & 12 & 20 & \begin{tabular}{|l|}
32 \\
\end{tabular} & \begin{tabular}{|l|l|}
58 \\
\end{tabular} & \begin{tabular}{|l|}
69 \\
\end{tabular} & 97 & 155 & Tsea & 194 & $224 / 2$ & 219 & 233 & 270 & \begin{tabular}{|l|l|}
304 \\
\end{tabular} & \begin{tabular}{|l|l|}
262 & 2 \\
\end{tabular} & \begin{tabular}{l|l}
2555 & 2 \\
\end{tabular} & \begin{tabular}{l|l|}
232 & 2 \\
\end{tabular} & \begin{tabular}{l|l}
208 & 1 \\
\end{tabular} & 169 & 16. 1 & 106 & \begin{tabular}{l|l}
83 & 5 \\
\end{tabular} & \begin{tabular}{l|l}
51 & 2 \\
\end{tabular} & \begin{tabular}{l|l}
29 & 2 \\
\end{tabular} & 25 & \begin{tabular}{l|l}
11 \\
\end{tabular} & 5 & 2 & 1 & & & 3637 \\
\hline & \begin{tabular}{|l|}
69 \\
\end{tabular} & & & \begin{tabular}{l|l}
1 \\
\end{tabular} & \begin{tabular}{|l|}
2 \\
\end{tabular} & $\begin{array}{ll}1 \\
\end{array}$ & 2 & 6 & \begin{tabular}{|l|}
7 \\
\end{tabular} & \begin{tabular}{|l|}
22 \\
\end{tabular} & 34 & 39 & 62 & \begin{tabular}{|l|}
74 \\
\end{tabular} & \begin{tabular}{|l|}
96 \\
\end{tabular} & 106 & $\pi+1$ & 156 & 170 & 171 & \begin{tabular}{ll|}
179 \\
\end{tabular} & \begin{tabular}{ll|}
181 & 1
\end{tabular} & $\begin{array}{lll}1699 & 1\end{array}$ & \begin{tabular}{l|l|}
186 & 1 \\
\end{tabular} & 158 & 1051 & 107 & \begin{tabular}{l|l}
96 \\
\end{tabular} & \begin{tabular}{l|l}
52 & 5
\end{tabular} & \begin{tabular}{l|l}
54 & 3 \\
\end{tabular} & \begin{tabular}{l|l}
31 & 2 \\
\end{tabular} & \begin{tabular}{l|l|}
23 & \\
\end{tabular} & 8 & 3 & 2 & 1 & & & 2428 \\
\hline & 71 & & & & & & 2 & 2 & 6 & \begin{tabular}{|l|l|}
4 \\
\end{tabular} & \begin{tabular}{|l|l|}
17 \\
\end{tabular} & \begin{tabular}{|l|}
13 \\
\end{tabular} & 25 & 24 & 44 & 52 & \begin{tabular}{l|l|}
74 \\
\end{tabular} & 81 & 101 & 119 & 113 & 7181 & $\overline{127} 1$ & $\begin{array}{ll}104 & 1 \\
\end{array}$ & 115 & \begin{tabular}{|l|}
91 \\
\end{tabular} & \begin{tabular}{l|l|}
84 & \\
\end{tabular} & \begin{tabular}{|l|l|}
58 & \\
\end{tabular} & \begin{tabular}{l|l}
54 & 2 \\
\end{tabular} & \begin{tabular}{l|l}
29 & 2 \\
\end{tabular} & \begin{tabular}{l|l}
22 & 1 \\
\end{tabular} & \begin{tabular}{l|l}
12 \\
\end{tabular} & 5 & 6 & 2 & 3 & & & 1507 \\
\hline & $73 \mid$ & & & & & & & 1 & 4 & 1 & 7 & 9 & 13 & 24 & 23 & 29 & \begin{tabular}{l|l}
37 \\
\end{tabular} & 36 & \begin{tabular}{|l|}
59 \\
\end{tabular} & \begin{tabular}{l|l|}
70 \\
\end{tabular} & \begin{tabular}{l|l|}
50 \\
\end{tabular} & 70 & \begin{tabular}{l|l}
65 \\
\end{tabular} & 60 & \begin{tabular}{l|l}
65 \\
\end{tabular} & \begin{tabular}{|l|l|}
49 \\
\end{tabular} & \begin{tabular}{l|l}
53 & \\
\end{tabular} & \begin{tabular}{l|l}
34 \\
\end{tabular} & \begin{tabular}{l|l}
35 & 2 \\
\end{tabular} & \begin{tabular}{l|l}
22 & 1 \\
\end{tabular} & 16 & \begin{tabular}{l|l}
13 \\
\end{tabular} & 3 & 2 & 2 & & & & 852 \\
\hline & 75 & & & & & & & & & $\begin{array}{ll} \\
\end{array}$ & 2 & 2 & 2 & 7 & \begin{tabular}{|l|}
11 \\
\end{tabular} & 14 & \begin{tabular}{l|l}
12 \\
\end{tabular} & 20 & \begin{tabular}{|l|}
25 \\
\end{tabular} & \begin{tabular}{l|l}
25 \\
\end{tabular} & \begin{tabular}{|l|}
33 \\
\end{tabular} & 39 & \begin{tabular}{l|l}
36 & \\
\end{tabular} & \begin{tabular}{l|l}
38 & \\
\end{tabular} & \begin{tabular}{l|l}
44 & \\
\end{tabular} & \begin{tabular}{|l|}
32 \\
\end{tabular} & \begin{tabular}{l|}
28 \\
\end{tabular} & \begin{tabular}{l|l|}
29 & \\
\end{tabular} & \begin{tabular}{l|l|}
20 \\
\end{tabular} & \begin{tabular}{l|l}
8 & 1 \\
\end{tabular} & 11 & 2 & 1 & & & & & & 442 \\
\hline & \begin{tabular}{|l|}
77 \\
\end{tabular} & & & & & & & 1 & & 2 & & \begin{tabular}{|l|}
1 \\
\end{tabular} & 1 & 1 & 5 & 4 & 5 & 6 & \begin{tabular}{|l|}
16 \\
\end{tabular} & 14 & $\begin{array}{ll}13 \\
\end{array}$ & \begin{tabular}{l|l}
15 & \\
\end{tabular} & \begin{tabular}{l|l}
12 \\
\end{tabular} & $\begin{array}{ll}17 \\
\end{array}$ & \begin{tabular}{l|l}
23 & \\
\end{tabular} & \begin{tabular}{|l|l|}
14 \\
\end{tabular} & \begin{tabular}{l|l|}
15 \\
\end{tabular} & \begin{tabular}{l|l}
11 \\
\end{tabular} & \begin{tabular}{l|l}
11 & 1 \\
\end{tabular} & 14 & 5 & 1 & 5 & & 1 & & & & 213 \\
\hline & \begin{tabular}{|l|l|}
79 \\
\end{tabular} & & & & & & & & & & & 1 & & & \begin{tabular}{|l|}
2 \\
\end{tabular} & 1 & 1 & 7 & 2 & 5 & 5 & 5 & 9 & 10 & 6 & \begin{tabular}{|l|l|}
7 \\
\end{tabular} & \begin{tabular}{l|l|}
12 & \\
\end{tabular} & 8 & 4 & 3 & 5 & 5 & 3 & & 1 & & & & 102 \\
\hline & 81 & & & & & & & & & & & & & & & 2 & 1 & 1 & \begin{tabular}{|l|l|}
1 \\
\end{tabular} & 2 & 4 & 2 & \begin{tabular}{l|l}
44 \\
\end{tabular} & 2 & 4 & 5 & 3 & 9 & 3 & \begin{tabular}{l|l}
2 & 2 \\
\end{tabular} & 2 & 1 & & 2 & 1 & & & 1 & 52 \\
\hline & \begin{tabular}{|l|}
83 \\
\end{tabular} & & & & & & & & & & & & & & & & & & \begin{tabular}{|l|l}
1 \\
\end{tabular} & 3 & & & & 1 & 1 & 1 & & 1 & 2 & & & 1 & & & & & & & 11 \\
\hline & 85 & & & & & & & & & & & & & & & & & & & & & 1 & & & 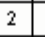 & & & & 2 & & & 1 & & & & & & & 6 \\
\hline & $87 \mid$ & & & & & & & & & & & & & & & & & & & & & & & & & & & & & & & & & & & & & & 0 \\
\hline & \begin{tabular}{|l|}
89 \\
\end{tabular} & & & & & & & & & & & & & & & & & & & & & & & & & & & & 1 & & & & & & & & & & 1 \\
\hline & tal & & & - & 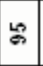 & & $\frac{T}{m}$ & 9 & 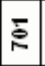 & 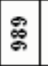 & ] & 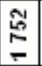 & 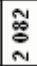 & 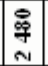 & 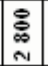 & $\begin{array}{l}\infty \\
\dot{m} \\
\end{array}$ & 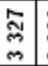 & 怨 & \begin{tabular}{l|}
$\mathscr{m}$ \\
$\bar{g}$ \\
$\bar{m}$
\end{tabular} & $\frac{F}{7}$ & ल⿸户 & $\begin{array}{ll}\circ \\
\end{array}$ & $\begin{array}{l}\infty \\
\stackrel{2}{0} \\
\text { I }\end{array}$ & $\begin{array}{l}\stackrel{8}{q} \\
\text { al } \\
\mathrm{N}\end{array}$ & 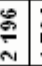 & \begin{tabular}{l} 
离 \\
\hdashline \\
\end{tabular} & $\stackrel{0}{\leftarrow}$ & $\stackrel{2}{\circ}$ & 心 & 鱼 & है & $\div$ & $\infty$ & m & & 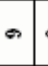 & 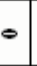 & & 50000 \\
\hline
\end{tabular}

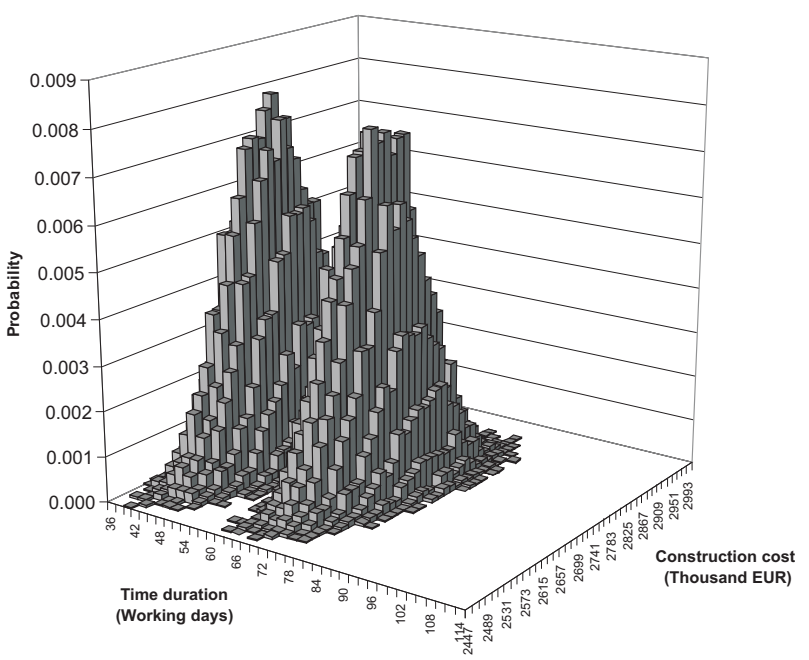

Fig. 5: Example of a 3D probability bar chart with heterogeneous solutions

of 21 days. After that period, the ground works can be resumed.

This specific condition leads to a heterogeneous solution of the simulation. It is difficult to find the solution of this building project using standard statistical methods. It is convenient to take advantage of visualization techniques and particular simulation calculations.

Important information regarding a proposal for a future project time schedule is specified by tests of the potential scenarios of the project development with current fixing of certain parameters of the building organizational model. Important information can be obtained about critical parameters of the planned project, for example by fixing the

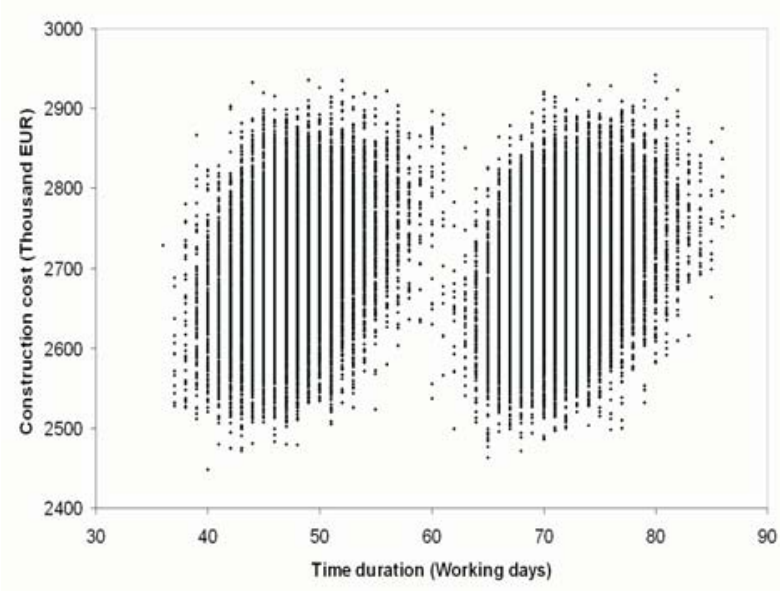

Fig. 5a: Example of a graph with interdependences between time duration and construction cost (frequencies)

deviations of the scope of the work for particular activities, see $\boldsymbol{Q}$ in expression (1) [7].

It is common practice to present the probability of the total construction time of a building project without the cost viewpoint (Fig. 4). A better-expressed project cost is presented as a respected fixed value that will be stable and independent from the duration of the project. Addressing this notion, the proposed approach that simulates the interrelated time and cost values shown in Fig. 3, is more understandable and more comprehensive than the information shown in Fig. 4, where

$$
\sum_{T} \mathbf{P}(a \leq T \leq b)=F\left(t_{b}\right)-F\left(t_{a}\right)
$$


or than the calculation with a fixed scope of work

$\sum_{T} \mathbf{P}(45 \leq T \leq 89)=\mathbf{P}\left(\operatorname{sim} \boldsymbol{T} \boldsymbol{A} \boldsymbol{B}_{\text {project }}(T)\right)$.

A similar situation arises if we fix the alternation of time schedules for the project. The scope of the work given as $C$ is specified as

$\sum_{C} \mathbf{P}(x \leq T \leq y)=F\left(c_{y}\right)-F\left(c_{x}\right)$

for the data simulated in Table 2 and demonstrated in Fig. 4.

$\sum_{C} \mathbf{P}(2411 \leq C \leq 3131)=\mathbf{P}\left(\operatorname{sim} \boldsymbol{T} \boldsymbol{A} \boldsymbol{B}_{\text {project }}(C)\right)$,

in the calculation with a fixed time ratio.

Fig. 4 shows the changes in the project cost with fixed duration of the observed project.

The expected time duration of the total construction project is given by its mean value

$\mathbf{E}\left[T_{\text {project }} \mid C=\right.$ const. $]=\sum_{T} t_{i} P\left(T=t_{i}\right)=\bar{t}$

In the same way, we can quantify the expected scope of the work on the total construction project by its mean value

$\mathbf{E}\left[C_{\text {project }} \mid T=\right.$ const. $]=\sum_{C} c_{i} P\left(C=c_{i}\right)=\bar{c}$

\section{The search for reliable construction cost and time duration}

The simulation model is able to calculate the adequate construction costs and the time duration of a project on the basis of the input probability level. The reciprocal view aims to find out the adequate level of probability for construction cost and activity durations.

There are two ways to calculate an adequate level of probability. The first way consists in fixing one variable parameter and investigating the changes in the remaining parameter. The second way involves a simultaneous investigation of the deviations of both parameters.

The approach used in this paper is based on expression (1) and Table 3 (discrete probability density table), and it enables us according to the data in Table 2 to calculate the level of probability as a cumulative density function

$$
F(T ; C)=\sum_{T} \sum_{C} P\left(T=t_{i} ; C=c_{i}\right),
$$

where $t_{i}$ and $c_{i}$ run through the set of all possible values of $T$ and $C$ and

$$
\sum_{T} \sum_{C} P\left(T=t_{i} ; C=c_{i}\right)=1 .
$$

In the course of a closer investigation of the results of particular simulations, the dependence between the level of probability and the construction cost and time duration was found. The following figures show the bilateral interactions of these project parameters.

\section{Conclusion}

The model described here enables us to predict the expected costs and the duration schedule of a project depending on input parameters such as the production rate, the scope of the work, the time schedule, bonding conditions, maximum and minimum deviations from the scope of the work, and the production rate. The results present a useful risk evaluation for projects or project activities.

\begin{tabular}{|c|c|c|c|c|c|c|c|c|c|c|c|c|c|c|c|c|c|c|c|c|c|c|c|c|c|c|c|c|c|c|c|c|c|c|c|c|c|c|c|c|c|}
\hline \multirow{2}{*}{\multicolumn{2}{|c|}{ Classes }} & \multicolumn{39}{|c|}{ Scope of work (Thousand EUR) } & \multirow[b]{2}{*}{ Total } \\
\hline & & $\sum^{\frac{2}{2}}$ & มั & กั & 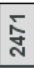 & ปั & זัָ & 於 & 商 & $\overline{\hat{N}^{2}}$ & 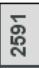 & $\sum_{\grave{c}}$ & స్త్ర & స్⿳亠े & 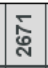 & 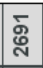 & ก̀ & กั & ลั & ลิ & ลั & 离 & 怘 & is & & 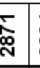 & 㞼 & হั) & న్ & זूু & ลิ & হু & Г্ল & ह్ల & Бू & $\Sigma$ & हृ & & E & $\frac{\bar{m}}{m}$ & \\
\hline \multirow{23}{*}{ 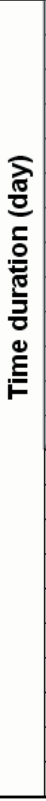 } & 45 & & & & & 1 & & & 1 & & & & & & 1 & & & 1 & & & 1 & & & & & & & & & & & & & & & & & & & & 5 \\
\hline & 47 & & & & & 2 & 4 & 1 & 1 & 5 & 1 & 2 & 1 & 1 & 2 & 2 & 1 & 1 & & & & & & & & 2 & & & & & & & & & & & & & & & 26 \\
\hline & 49 & & 1 & 2 & 4 & 6 & 10 & 6 & 14 & 17 & 17 & 19 & 19 & 25 & 18 & 20 & 21 & 14 & 8 & 13 & 7 & 5 & 5 & 3 & 3 & 2 & 1 & 1 & & & & & & & & & & & & & 258 \\
\hline & 51 & 1 & 3 & 5 & 8 & 19 & 23 & 33 & 42 & 42 & 68 & 68 & 71 & 74 & 69 & 81 & 67 & 41 & 54 & 60 & 30 & 31 & 19 & 10 & \begin{tabular}{l|l}
0 & \\
\end{tabular} & 7 & 9 & 2 & & 1 & 2 & & & & & & & & & & 940 \\
\hline & 53 & 2 & 3 & 6 & 10 & 24 & 41 & 64 & 81 & \multirow{2}{*}{\multicolumn{7}{|c|}{ Level of probability: }} & 158 & 145 & 122 & 94 & 113 & \begin{tabular}{|l|l|}
3 & 84 \\
\end{tabular} & 86 & 5 & \begin{tabular}{l|l}
6 & 4
\end{tabular} & \begin{tabular}{l|l}
43 \\
\end{tabular} & 30 & 16 & 8 & 8 & & 2 & & 1 & & & & & & & 2270 \\
\hline & 55 & 1 & 5 & 2 & 13 & 34 & 53 & 77 & & & & & & & & & 36 & 307 & 287 & 267 & 25 & 320 & \begin{tabular}{l|l|}
5 & 17 \\
\end{tabular} & 712 & \begin{tabular}{l|l}
27 & 1 \\
\end{tabular} & 27 & 75 & 56 & 34 & 23 & 10 & 6 & 5 & & & 2 & & & & & 4386 \\
\hline & 57 & 1 & & 8 & 20 & 26 & 63 & 87 & & \multicolumn{7}{|c|}{0,7476} & 154 & 478 & 477 & 458 & 38 & \begin{tabular}{|l|l|}
334 \\
\end{tabular} & 128 & 626 & $63 \sqrt{2}$ & $4 \sqrt{1}$ & 141 & 111 & 87 & 52 & 26 & 23 & 9 & 3 & 2 & 1 & & & & & 6457 \\
\hline & 59 & & 1 & 2 & 12 & 28 & 46 & 87 & 110 & 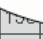 & & & & & & 480 & 535 & 543 & 552 & 542 & 465 & 90 & $\begin{array}{ll}2 & 39 \\
\end{array}$ & 735 & \begin{tabular}{l|l}
512 \\
\end{tabular} & 2942 & 250 & 174 & 129 & 92 & 58 & 31 & 17 & 10 & 6 & 2 & 1 & & & & 7516 \\
\hline & 61 & & & 4 & 9 & 21 & 33 & 50 & 98 & 140 & 212 & 257 & 313 & 319 & 410 & 491 & 514 & 485 & 522 & 515 & 520 & 48 & 043 & 438 & \begin{tabular}{l|l}
82 & 2 \\
\end{tabular} & 298 & 252 & 197 & 141 & 87 & 60 & 28 & 22 & 10 & 3 & 1 & 2 & & & & 7310 \\
\hline & 63 & & 1 & 1 & 8 & 10 & 23 & 47 & 56 & 93 & 137 & 178 & 230 & 279 & 355 & 378 & 465 & 447 & 445 & 462 & 2480 & 41 & \begin{tabular}{|l|l|}
5 & 38 \\
\end{tabular} & 334 & \begin{tabular}{l|l}
48 & 3 \\
\end{tabular} & $315 \mid 2$ & 278 & 228 & 170 & 112 & 83 & 53 & 32 & 15 & 2 & 2 & & & & 1 & 6532 \\
\hline & 65 & & 1 & & 6 & 8 & 10 & 22 & 40 & 56 & 106 & 137 & 130 & 210 & 259 & 251 & 308 & 341 & 364 & 351 & 37 & \begin{tabular}{l|l}
4 & 35 \\
\end{tabular} & \begin{tabular}{|l|l|}
3 & 33 \\
\end{tabular} & $\begin{array}{ll}429 \\
\end{array}$ & \begin{tabular}{l|l}
90 & 2 \\
\end{tabular} & 2772 & 245 & 168 & 154 & 97 & 65 & 41 & 22 & 13 & 12 & 3 & 1 & & & & 5049 \\
\hline & 67 & & & 1 & 3 & 3 & 4 & 12 & 20 & 32 & 58 & 69 & 97 & 155 & 139 & 194 & 224 & 219 & 233 & 270 & $30<$ & 426 & $\begin{array}{ll}2 & 25 \\
\end{array}$ & 5 & \begin{tabular}{l|l|}
32 & 22 \\
\end{tabular} & \begin{tabular}{l|l}
208 & 1 \\
\end{tabular} & 169 & 161 & 106 & 83 & 51 & 29 & 25 & 11 & 5 & 2 & 1 & & & & 3637 \\
\hline & 69 & & & 1 & 2 & 1 & 2 & 6 & 7 & 22 & 34 & 39 & 62 & 74 & 96 & 106 & 114 & 156 & 170 & 171 & 179 & \begin{tabular}{l|l}
9 & 18 \\
\end{tabular} & \begin{tabular}{l|l}
1 & 16 \\
\end{tabular} & 918 & \begin{tabular}{l|l}
86 & 1 \\
\end{tabular} & \begin{tabular}{l|l}
158 & 1 \\
\end{tabular} & 115 & 107 & 96 & 52 & 54 & 31 & 23 & 8 & 3 & 2 & 1 & & & & 2428 \\
\hline & 71 & & & & & & 2 & 2 & 6 & 4 & 17 & 13 & 25 & 24 & 44 & 52 & 74 & 81 & 101 & 119 & 9113 & \begin{tabular}{|l|l|}
3 & 11 \\
\end{tabular} & \begin{tabular}{l|l|}
8 & 12 \\
\end{tabular} & \begin{tabular}{l|l|l|}
7 & 10 \\
\end{tabular} & \begin{tabular}{l|l}
94 & 1 \\
\end{tabular} & 15 & \begin{tabular}{l|l|}
91 &
\end{tabular} & 84 & 58 & 54 & 29 & 22 & 12 & 5 & 6 & 2 & 3 & & & & 1507 \\
\hline & 73 & & & & & & & 1 & 4 & 1 & 7 & 9 & 13 & 24 & 23 & 29 & 37 & 36 & 59 & 70 & 50 & 70 & 65 & \begin{tabular}{|l|l|}
5 & 60 \\
\end{tabular} & $\begin{array}{ll}0 \\
0\end{array}$ & 65 & \begin{tabular}{l|l}
49 \\
\end{tabular} & 53 & 34 & 35 & 22 & 16 & 13 & 3 & 2 & 2 & & & & & 852 \\
\hline & 75 & & & & & & & & & 1 & 2 & 2 & 2 & 7 & 11 & 14 & 12 & 20 & 25 & 25 & 33 & 39 & 36 & \begin{tabular}{|l|l|}
6 & 38
\end{tabular} & \begin{tabular}{l|l}
8 & 4
\end{tabular} & \begin{tabular}{l|l}
44 \\
\end{tabular} & \begin{tabular}{l|l}
32 & \\
\end{tabular} & 28 & 29 & 20 & 8 & 11 & 2 & 1 & & & & & & & 442 \\
\hline & 77 & & & & & & & 1 & & 2 & & 1 & 1 & 1 & 5 & 4 & 5 & 6 & 16 & 14 & 13 & 15 & \begin{tabular}{|l|l|}
512 \\
\end{tabular} & \begin{tabular}{l|l}
2 & 1 \\
\end{tabular} & 7 & 23 & \begin{tabular}{l|l}
14 &
\end{tabular} & 15 & 11 & 11 & 14 & 5 & 1 & 5 & & 1 & & & & & 213 \\
\hline & 79 & & & & & & & & & & & 1 & & & 2 & 1 & 1 & 7 & 2 & 5 & 5 & 5 & 9 & 10 & 0 & 6 & 7 & 12 & 8 & 4 & 3 & 5 & 5 & 3 & & 1 & & & & & 102 \\
\hline & 81 & & & & & & & & & & 1 & 7 & & & & 2 & 1 & 1 & 1 & 2 & 4 & 2 & 4 & 2 & 2 & 4 & 5 & 3 & 9 & 3 & 2 & 2 & 1 & & 2 & 1 & & & & 1 & 52 \\
\hline & 83 & & & & & & & & & & & & & & & & & & 1 & 3 & & & & 1 & 1 & 1 & 1 & & 1 & 2 & & & 1 & & & & & & & & 11 \\
\hline & 85 & & & & & & & & & & & & & & & & & & & & & 1 & & & & 2 & & & & 2 & & & 1 & & & & & & & & 6 \\
\hline & 87 & & & & & & & & & & & & & & & & & & & & & & & & & & & & & & & & & & & & & & & & 0 \\
\hline & 89 & & & & & & & & & & & & & & & & & & & & & & & & & & & & & 1 & & & & & & & & & & & 1 \\
\hline \multicolumn{2}{|c|}{ Total } & 10 & ю & న్ల & Һ & జ్ & $\frac{\nabla}{m}$ & 过 & $\bar{R}$ & $\stackrel{\Re}{\mathscr{乃}}$ & 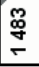 & 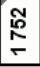 & 芯 & 兽 & $\begin{array}{c}\stackrel{8}{0} \\
\text { N } \\
\end{array}$ & $\begin{array}{l}0 \\
0 \\
\text { m }\end{array}$ & స్ల్ల & 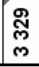 & 总 & $\underset{m}{j}$ & 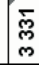 & $\begin{array}{l}8 \\
0 \\
m\end{array}$ & 怘 & 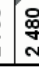 & & 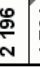 & 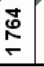 & 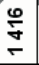 & $\begin{array}{l}\text { م } \\
\stackrel{0}{\circ} \\
\end{array}$ & 尺̊. & 敦 & 일 & 市 & ® & \% & ส & $\sigma$ & & o & $N$ & 50000 \\
\hline
\end{tabular}

Table 3: Example of calculating the level of Discrete Probability Distribution (DPD) for 50000 simulations 


\section{Acknowledgement}

This paper was developed with financial support from the Ministry of Education, Youth and Sports of the Czech Republic, project No. 1M6840770001.

\section{References}

[1] Beran, V., Dlask, P.: Management udržitelného rozvoje. Praha: Academia, 2005.

[2] Beran, V., Dlask, P., Heralová, R., Berka, V.: Dynamický harmonogram, rozurhováni výroby do času. 1. vyd. Praha: Academia, 2002. 170 s. ISBN 80-200-1007-6.

[3] Dlask, P., Beran, V.: MDM 2004 - teoretická př́ručka. 1. vyd. Praha: Vydavatelství ČVUT, 2004. 90 s. ISBN 80-01-03072-5.

[4] Haas, Š., Hájek, V.: Systémové plánováni a ř́zení ve stavebnictví. Praha: SNTL, 1981.

[5] Heralová, R., Frková, J., Tománková, J.: Decision Making and Bids in Construction Industry. Integrated Risk Management. Berlin: Humbolt Universität Berlin, 2002.
[6] Hromada, E., Beran, V.: Reliable Construction Cost and Time Estimation on the basis of the Dynamic Flow-Chart (Software for Project Reliability Estimation and Risk Evaluation). In: Advanced Engineering Design AED 2006 [CD-ROM]. Prague: CTU, 2006, vol. 1, p. P3.14-1-P3.14-15. ISBN 80-86059-44-8.

[7] Hromada, E., Beran, V.: Management časových a finančních potřeb stavebních zakázek. In: Stavebnictví a interiér. 2005, roč. 2005, č. 12, s. 98-100. ISSN 1211-6017.

Doc. Ing. Václav Beran, DrSc.

phone: +4202 24353720

Fax: +420224355439

e-mail: beran@fsv.cvut.cz

Ing. Eduard Hromada, Ph.D.

e-mail: eduard.hromada@fsv.cvut.cz

Czech Technical University in Prague

Faculty of Civil Engineering

Thákurova 7

16629 Praha 6, Czech Republic 